\title{
PIEZOELECTRIC BEAM FINITE ELEMENT MODEL AND ITS REDUCTION TO STATE-SPACE MODEL
}

\author{
VLADIMÍR KUTIŠ*, JURAJ PAULECH*, GABRIEL GÁLIK*, \\ JUSTÍN MURÍN* AND VLADIMÍR GOGA* \\ *Institute of Automotive Mechatronics, \\ Faculty of Electrical Engineering and Information Technology, \\ Slovak University of Technology in Bratislava, \\ Ilkovičova 3, 81219 Bratislava 1, \\ Slovak Republic. \\ e-mail: vladimir.kutis@stuba.sk, web page: http://uamt.fei.stuba.sk/web/
}

Key words: Finite Element Method, Piezoelectric Analysis, State-Space Model, LQR Control, MOR

\begin{abstract}
The paper deals with modeling and simulation of the connection of piezoelectric materials, FGM materials and LQR control, using state-space model, which is built by modal truncation MOR method. FEM equations of piezoelectric finite beam were implemented in FEM program MultiFEM written in Wolfram Language. Modal truncation MOR method, which uses results from modal analysis, is also implemented in program Wolfram Mathematica. Simple piezoelectric structure is analyzed in numerical experiments, where FEM results are compared with MOR results.
\end{abstract}

\section{INTRODUCTION}

Current mechatronic systems use a combination of smart materials [1] and modern control [2]. Modern smart materials include magnetorheological materials, functionally graded materials, piezoelectric materials, shape memory materials and many more [3]. Modern control methods use a state description of the system's behavior and these control methods include linear quadratic controller (LQR) and linear quadratic Gaussian controller (LQG) [4]. To model a physical system, one of the most effective methods is the finite element method [5], which can describe the analyzed system with high accuracy. However, this accuracy will be reflected in an increase the number of degrees of freedom, which is undesirable from the control viewpoint. Therefore, the FEM model must be reduced to decrease the number of degrees of freedom. For this purpose, the modal truncation method, which is one of model order reduction (MOR) methods [6], is used. 
This paper deals with modeling and simulation of the connection of piezoelectric materials, FGM materials and LQR control, using state-space model, which is built by modal truncation MOR method. In the theoretical part of the paper, the FEM equations of a piezoelectric beam element with an FGM core is presented, then a reduced state-space model is described, which uses the modal truncation method and at the end of the theoretical part the LQR controller is described. The derived relations were implemented into the FEM program MultiFEM, while the built-in functions of the Wolfram Mathematica program [7] are used to formulate the state-space model. The functionality of the system is presented on a simple example of a beam with an FGM core and piezoelectric layers, which is analyzed using the FEM program MultiFEM and then reduced and analyzed as a state-space model.

\section{PIEZOELECTRIC BEAM STRUCTURE}

Fig. 1 shows a two-node beam finite element, the core of beam is made of FGM material, piezoelectric layers with electrodes are placed on the outer surfaces of beam core. The length of the beam finite element is $L^{e}$, the width is $b^{e}$, the height FGM of the
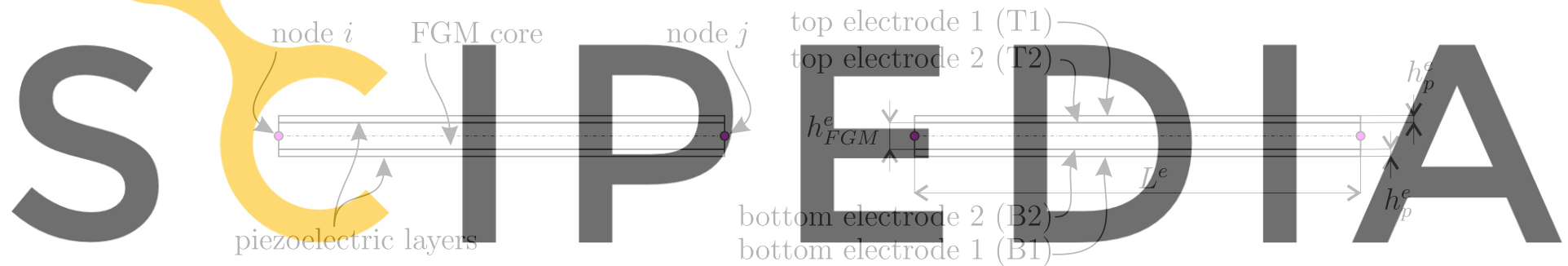

Register for free at https//www.scipedia.com to download the version without the watermark

Figure 1: Beam element with FGM core and piezoelectric layers with electordes

core is $h_{F G M}^{e}$ and the height of one piezoelectric layer is $h_{p}^{e}$. This finite element has 10 degrees of freedom -6 mechanical and 4 electrical, which are shown in Fig. 2. Vector of
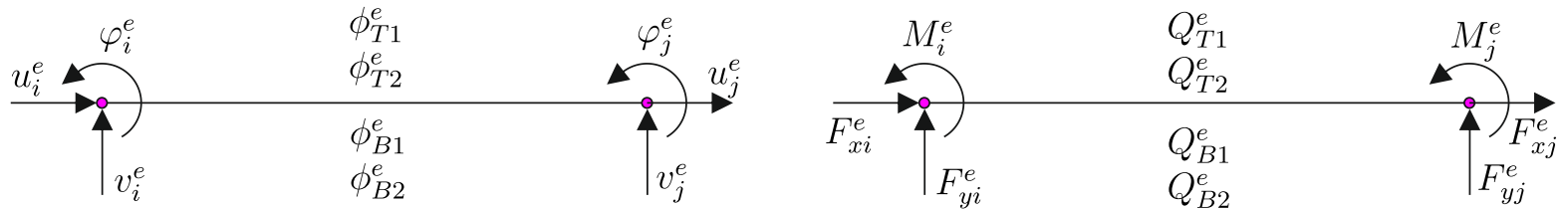

Figure 2: Piezoelectric beam element, degrees of freedom - left, element loads - right

element unknowns is defined as

$$
\left[\begin{array}{c}
\mathbf{v}^{e} \\
\phi^{e}
\end{array}\right]=\left[\begin{array}{llllllllll}
u_{i}^{e} & v_{i}^{e} & \varphi_{i}^{e} & u_{j}^{e} & v_{j}^{e} & \varphi_{j}^{e} & \phi_{T 1}^{e} & \phi_{T 2}^{e} & \phi_{B 1}^{e} & \phi_{B 2}^{e}
\end{array}\right]^{T}
$$


and vector of element loads is defined as

$$
\left[\begin{array}{l}
\mathbf{F}^{e} \\
\mathbf{Q}^{e}
\end{array}\right]=\left[\begin{array}{llllllllll}
F_{x i}^{e} & F_{y i}^{e} & M_{i}^{e} & F_{x j}^{e} & F_{y j}^{e} & M_{j}^{e} & Q_{T 1}^{e} & Q_{T 2}^{e} & Q_{B 1}^{e} & Q_{B 2}^{e}
\end{array}\right]^{T}
$$

FEM equations for beam element with piezoelectric layers and FGM core for transient analysis have classical form

$$
\left[\begin{array}{cc}
\mathbf{M}_{v v}^{e} & \mathbf{0} \\
\mathbf{0} & \mathbf{0}
\end{array}\right]\left[\begin{array}{l}
\ddot{\mathbf{v}}^{e} \\
\ddot{\boldsymbol{\phi}}^{e}
\end{array}\right]+\left[\begin{array}{cc}
\mathbf{C}_{v v}^{e} & \mathbf{0} \\
\mathbf{0} & \mathbf{0}
\end{array}\right]\left[\begin{array}{c}
\dot{\mathbf{v}}^{e} \\
\dot{\boldsymbol{\phi}}^{e}
\end{array}\right]+\left[\begin{array}{ll}
\mathbf{K}_{v v}^{e} & \mathbf{K}_{v \phi}^{e} \\
\mathbf{K}_{\phi v}^{e} & \mathbf{K}_{\phi \phi}^{e}
\end{array}\right]\left[\begin{array}{c}
\mathbf{v}^{e} \\
\boldsymbol{\phi}^{e}
\end{array}\right]=\left[\begin{array}{c}
\mathbf{F}^{e} \\
\mathbf{Q}^{e}
\end{array}\right]
$$

The piezoelectric system is described by the final FEM equations in the form

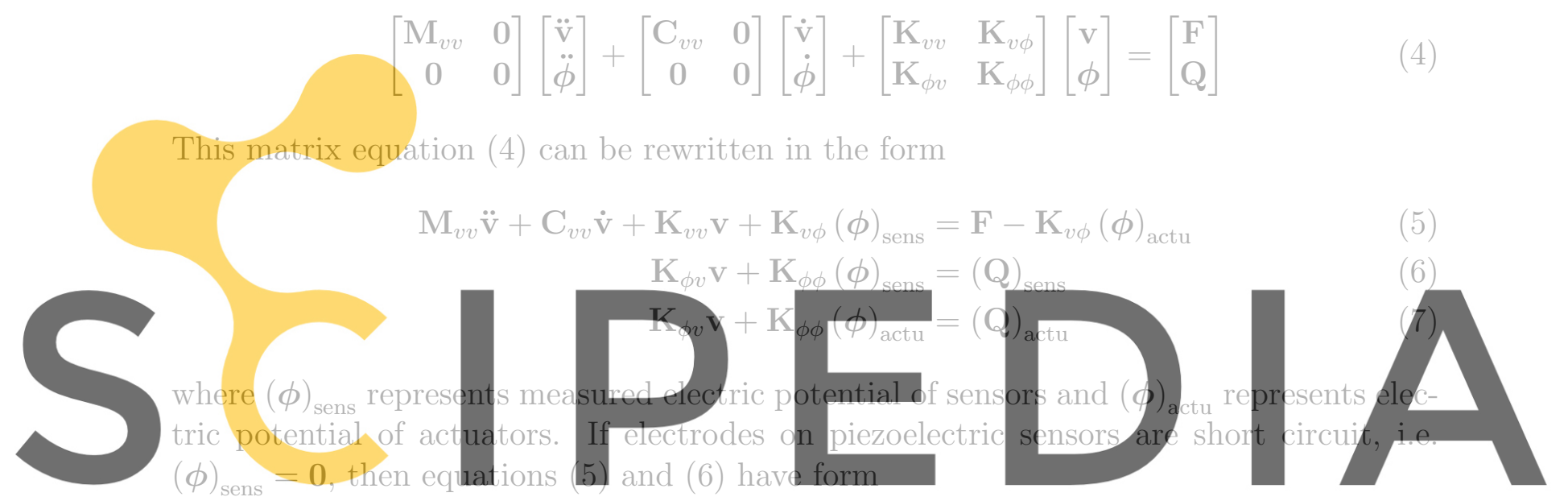

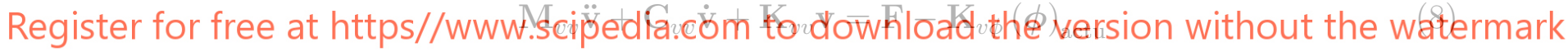

$$
\mathbb{K}_{\phi v} \mathbf{V}=(Q)_{\text {sens }}
$$

If electrodes on piezoelectric sensors are open circuit, i.e. $\mathbf{D}=\mathbf{0}$, then these equations have form

$$
\begin{aligned}
\mathbf{M}_{v v} \ddot{\mathbf{v}}+\mathbf{C}_{v v} \dot{\mathbf{v}}+\mathbf{K}_{v v} \mathbf{v}+\mathbf{K}_{v \phi}(\phi)_{\text {sens }} & =\mathbf{F}-\mathbf{K}_{v \phi}(\phi)_{\mathrm{actu}} \\
\mathbf{K}_{\phi v} \mathbf{v}+\mathbf{K}_{\phi \phi}(\boldsymbol{\phi})_{\mathrm{sens}} & =\mathbf{0}
\end{aligned}
$$

\section{STATE-SPACE MODEL}

The displacement of the nodal points expressed by the vector $\mathbf{v}$ can be expressed by the eigenshapes $\mathbf{Z}$ of the system and the amplitudes $\mathbf{x}$ of these eigenshapes, i.e. $\mathbf{v}=\mathbf{Z} . \mathbf{x}$. Using this transformation, equation (4) can be rewritten in form

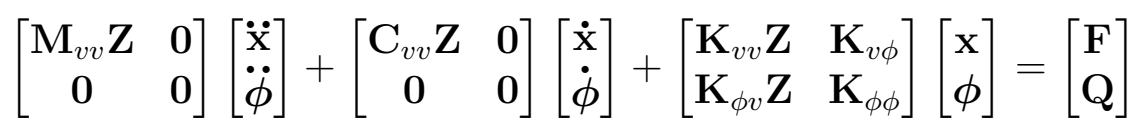


Using orthogonality properties of mode shapes

$$
\mathbf{Z}^{\mathrm{T}} \mathbf{M}_{v v} \mathbf{Z}=\operatorname{diag}\left(\mu_{k}\right) \quad \mathbf{Z}^{\mathrm{T}} \mathbf{K}_{v v} \mathbf{Z}=\operatorname{diag}\left(\mu_{k} \omega_{k}^{2}\right) \quad \mathbf{Z}^{\mathrm{T}} \mathbf{C}_{v v} \mathbf{Z}=\operatorname{diag}\left(2 \xi_{k} \mu_{k} \omega_{k}\right)
$$

equation (12) can be rewritten in form

$$
\left[\begin{array}{ll}
\boldsymbol{\mu} & \mathbf{0} \\
\mathbf{0} & \mathbf{0}
\end{array}\right]\left[\begin{array}{c}
\ddot{\mathbf{x}} \\
\ddot{\boldsymbol{\phi}}
\end{array}\right]+\left[\begin{array}{cc}
2 \boldsymbol{\xi} \boldsymbol{\mu} \boldsymbol{\omega} & \mathbf{0} \\
\mathbf{0} & \mathbf{0}
\end{array}\right]\left[\begin{array}{c}
\dot{\mathbf{x}} \\
\dot{\boldsymbol{\phi}}
\end{array}\right]+\left[\begin{array}{cc}
\boldsymbol{\mu} \boldsymbol{\omega}^{2} & \mathbf{Z}^{\mathrm{T}} \mathbf{K}_{v \phi} \\
\mathbf{K}_{\phi v} \mathbf{Z} & \mathbf{K}_{\phi \phi}
\end{array}\right]\left[\begin{array}{c}
\mathbf{x} \\
\phi
\end{array}\right]=\left[\begin{array}{c}
\mathbf{Z}^{\mathrm{T}} \mathbf{F} \\
\mathbf{Q}
\end{array}\right]
$$

where $\boldsymbol{\mu}, \boldsymbol{\omega}$ and $\boldsymbol{\xi}$ represent matrix of modal masses, matrix of modal frequencies and matrix of modal damping ratios of structure, respectively. After some manipulation, we can from equation (14) obtain state-space model

$$
\left[\begin{array}{l}
\dot{x} \\
\ddot{\mathbf{x}}
\end{array}\right]=\left[\begin{array}{cc}
0 & \underline{I} \\
-\omega^{2} & -2 \xi \omega
\end{array}\right]\left[\begin{array}{l}
\mathbf{x} \\
\dot{x}
\end{array}\right]+\left[\begin{array}{cc}
0 & 0 \\
\mu^{-1} \mathbf{Z}^{T} & -\mu^{-1} \mathbf{Z}^{T} \mathbb{K}_{v \phi}
\end{array}\right]\left[\begin{array}{l}
\mathbb{F} \\
\phi
\end{array}\right]
$$

\section{CONTROL OF MOR MODEL}

Linear quadratic controller is very suitable for control of flexible structural systems. Control law can be written as follows
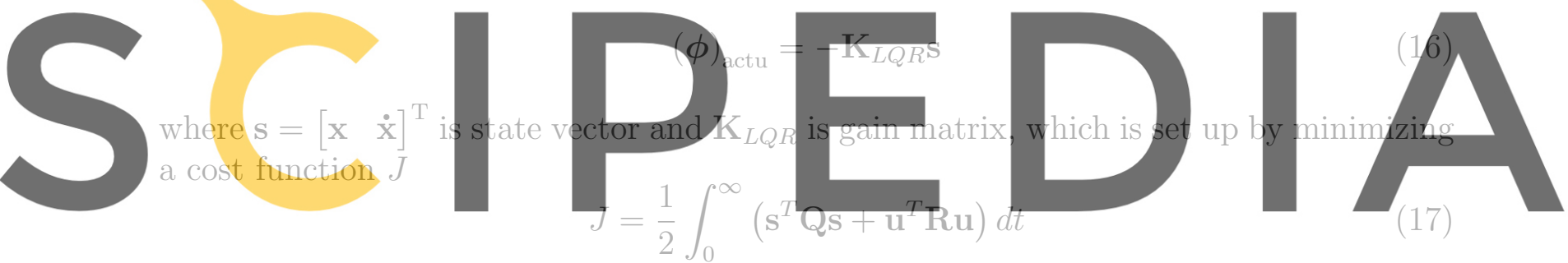

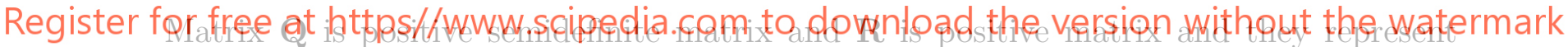

weights of state and input vectors in the process of minimization of a cost function $J$. Vector $\mathbf{u}=(\phi)_{\text {actu }}$ represents input vector.

\section{NUMERICAL EXAMPLE}

\subsection{Analyzed system}

Fig. 3 left shows the analyzed FGM beam structure on which the piezoelectric actuators and sensors are placed. The discretized FEM model is shown in Fig. 3 right. Effective material properties of FGM beam are defined by material properties of constituents and their variations and Young's modulus and density of considered FGM beam have form

$$
\begin{aligned}
E_{\mathrm{FGM}}(x, y)= & 1.9 \overline{3} \times 10^{19} x^{3} y^{2}-1.9 \overline{3} \times 10^{14} x^{3}-2.9 \times 10^{18} x^{2} y^{2}+ \\
& +2.9 \times 10^{13} x^{2}+5.8 \times 10^{15} y^{2}+2.55 \times 10^{11}[\mathrm{~Pa}] \\
\rho_{\mathrm{FGM}}(x, y)= & 1.34667 \times 10^{12} x^{3} y^{2}-1.34667 \times 10^{7} x^{3}-2.02 \times 10^{11} x^{2} y^{2}+ \\
& +2.02 \times 10^{6} x^{2}+4.04 \times 10^{8} y^{2}+9200 . \quad\left[\mathrm{kg} / \mathrm{m}^{3}\right]
\end{aligned}
$$




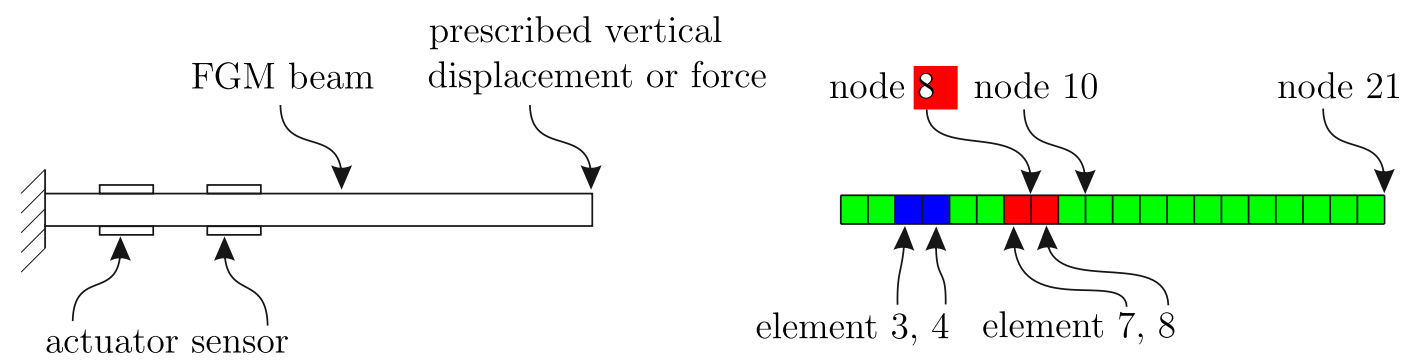

Figure 3: Analyzed FGM beam system with piezoelectric actuators and sensors - left, discretized FEM model - right

Damping of structure is considered as Rayleigh damping with mass and stiffness constants $3 \times 10^{-5} \mathrm{~s}^{-1}$ and $3 \times 10^{-5} \mathrm{~s}$, respectively.

Piezoelectric actuators and sensors are made from PZT5A piezoelectric material. PZT5A is orthotropic material and has following material properties (direction of poling has index 3):

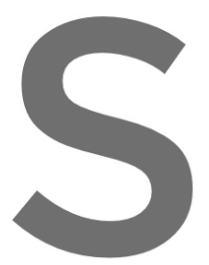

- mechanical properties:

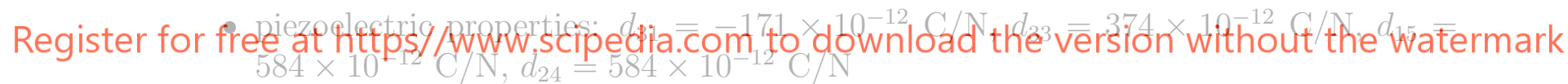

- relative permittivity: $\epsilon_{11}^{\sigma}=1728.8, \epsilon_{22}^{\sigma}=1728.8, \epsilon_{33}^{\sigma}=1694.9$

The goal of the analysis is to perform a static and dynamic FEM analysis of the beam structure with different excitations and then create a MOR model and compare the results obtained from the MOR model and the FEM model. The analysis also includes a design of LQR control of the structure in order to minimize the vibration of the structure.

\subsection{FEM analysis}

\section{Static analysis}

Static FEM analysis is considered as an initial analysis that defines the initial state for the selected transient analysis. The deformed state is defined by the prescribed vertical displacement at node 8 with a value of $-0.25 \times 10^{-5} \mathrm{~m}$ and at node 21 with a value of $1 \times 10^{-5} \mathrm{~m}-$ see Fig. 3. The deformed shape is shown in Fig. 4 . 


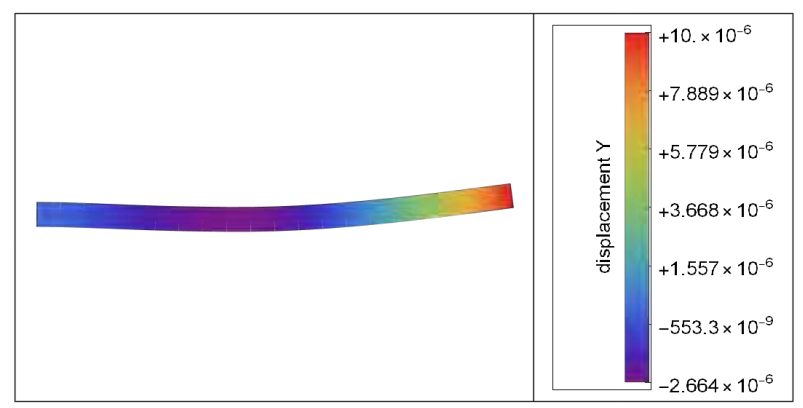

Figure 4: Static deformed shape of analyzed beam structure

\section{Modal analysis}

The goal of the modal analysis is to obtain the first 4 bending eigenfrequencies and the corresponding eigenmodes of the analyzed beam structure. These eigenfrequencies and eigenmodes are subsequently used both in the creation of a reduced model of the system and in the definition of the modal initial state of the system. The first four eigenshapes are shown in Fig. 5.

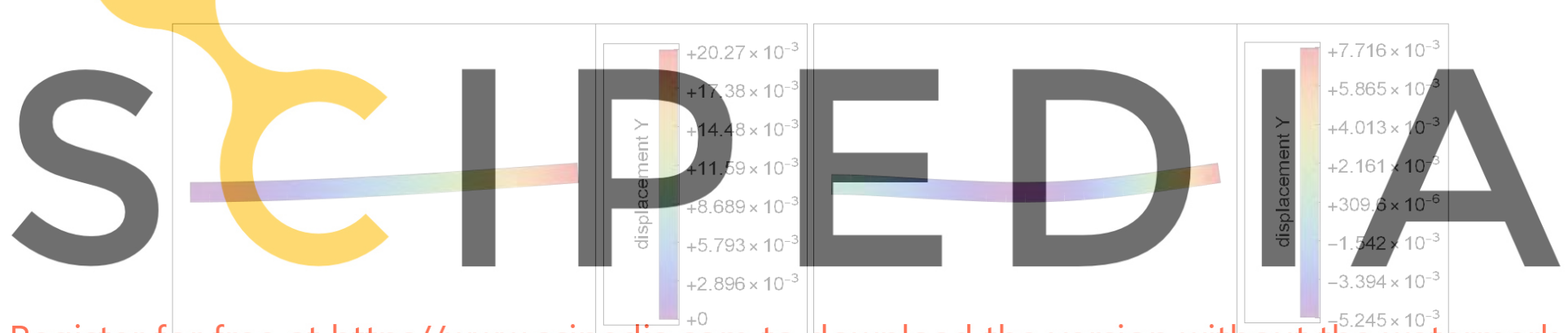

Register for free at https//Www.scipedia.com to download the version without the watermark

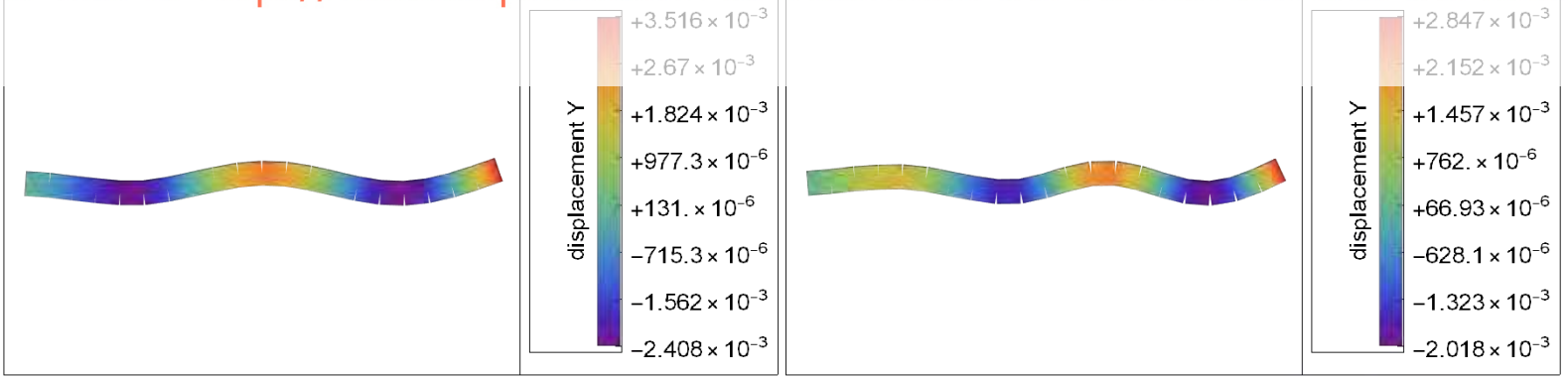

Figure 5: The first 4 bending eigenmodes

First four bending eigenfrequencies of analyzed systems are $188.4 \mathrm{~Hz}, 988.5 \mathrm{~Hz}, 5532 \mathrm{~Hz}$ and $9765 \mathrm{~Hz}$.

\section{Harmonic analysis}

The response of the analyzed structure to the harmonic vertical force acting at the end of the structure - node 21 is shown in Fig. 6. The amplitude of the harmonic force is 
20 N. As can be seen from the harmonic analysis of piezoelectric system with damping,

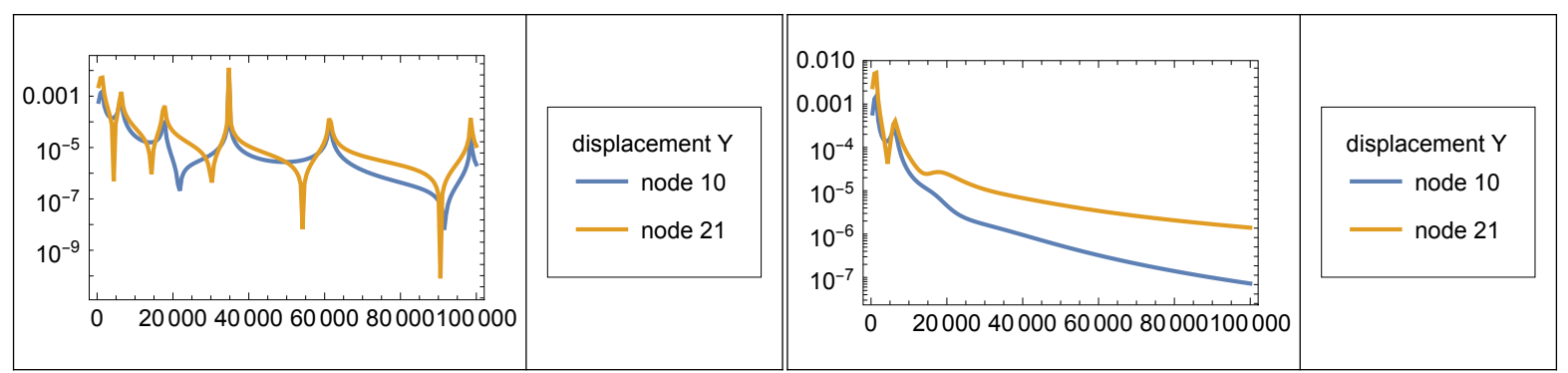

Figure 6: Response of system to harmonic vertical force, without considering damping - left, with damping - right

the dominant natural frequencies are the first four. For this reason, the first 4 bending eigenshapes and eigenfrequencies of the analyzed system are used in the reduced model.

Transient analysis

In the transient analysis, 3 different excitations were considered: excitation by initial conditions, excitation by external force, excitation by electric potential on actuators.

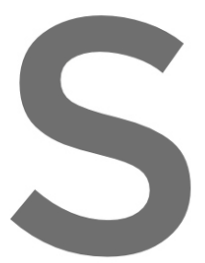

Excitation by initia
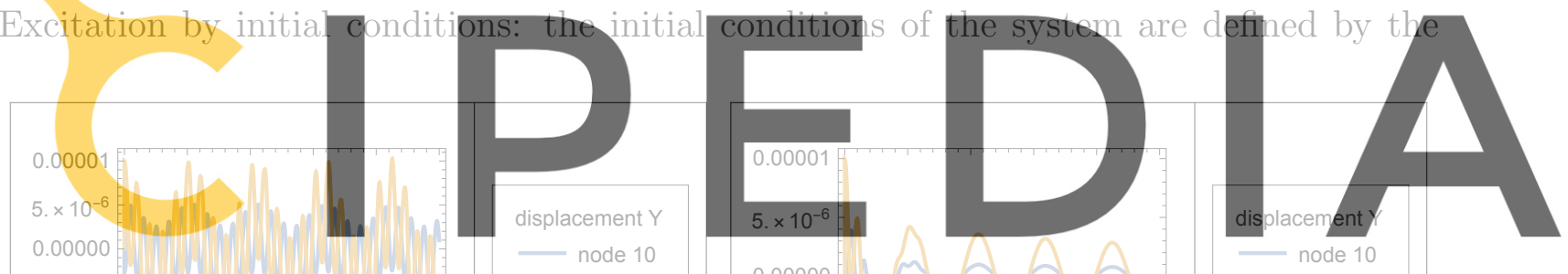

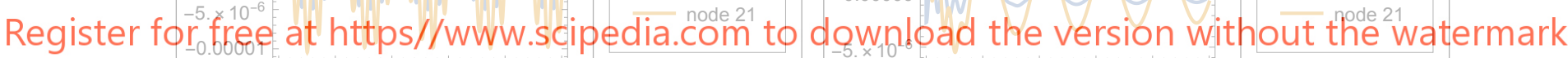
0.0000 .0050 .0100 .0150 .0200 .025
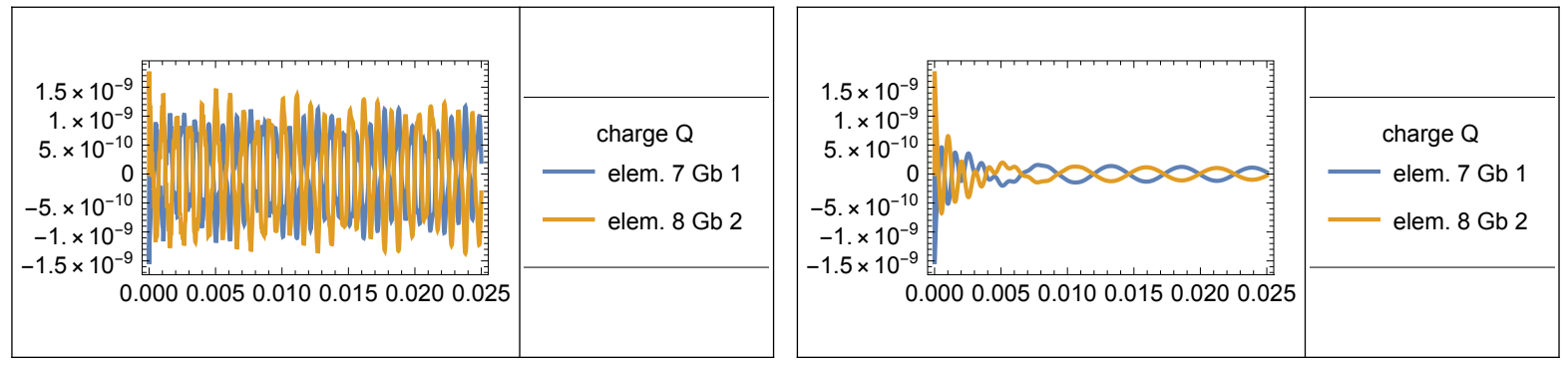

Figure 7: Excitation by initial conditions - displacements and the electric charge, without considering damping - left, with damping - right

static deformation, which was presented at the subsection Static analysis. Newmark's numerical method was used to calculate the system response. The displacements of the 
nodes 10 and 21 and the electric charge on the electrodes on the elements 7 and 8 are shown in Fig. 7.

Excitation by external force: the external force is located at the free end of the system node 21. The force has a harmonic character with frequency $80 \mathrm{~Hz}$ and an amplitude of $10 \mathrm{~N}$. The displacement of nodes 10 and 21 as well as the electric charge on elements 7 and 8 of damped system are shown in Fig. 8. The system had zero deformation and zero

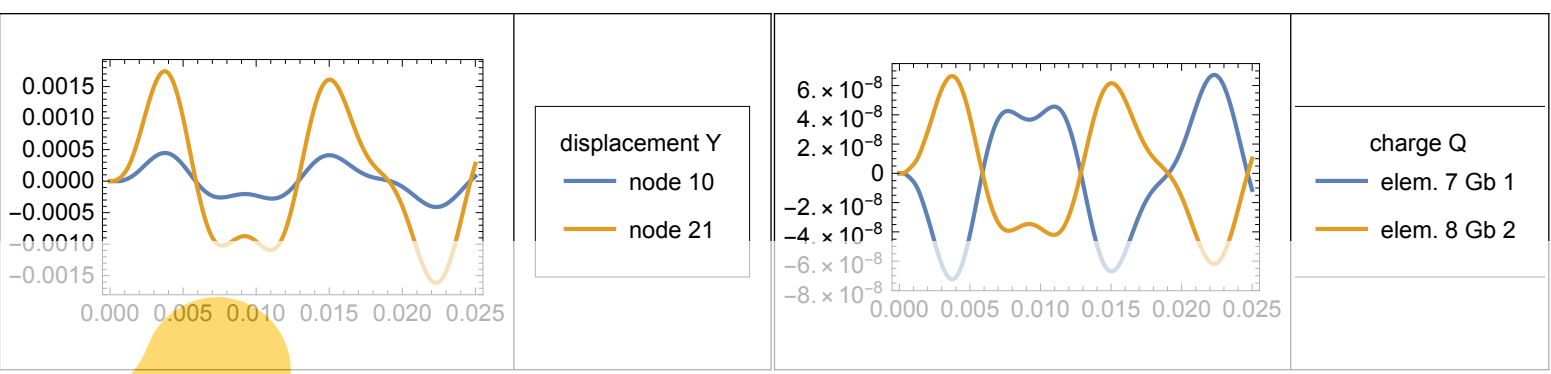

Figure 8: Excitation by external force - displacements and the electric charge

velocity at the beginning of investigated time.

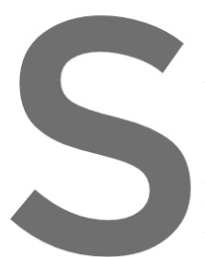

Excitation by electric

to the left side - elen

an electric harmonic

prescribed at the oute
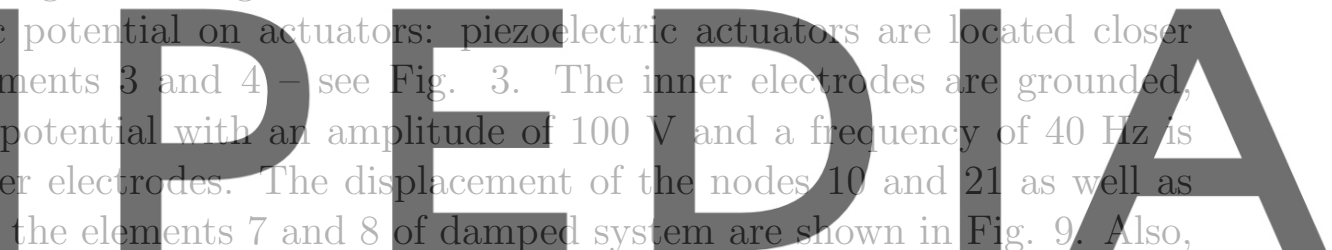

Register for free at https//www.scipedia.com to download the version without the watermark

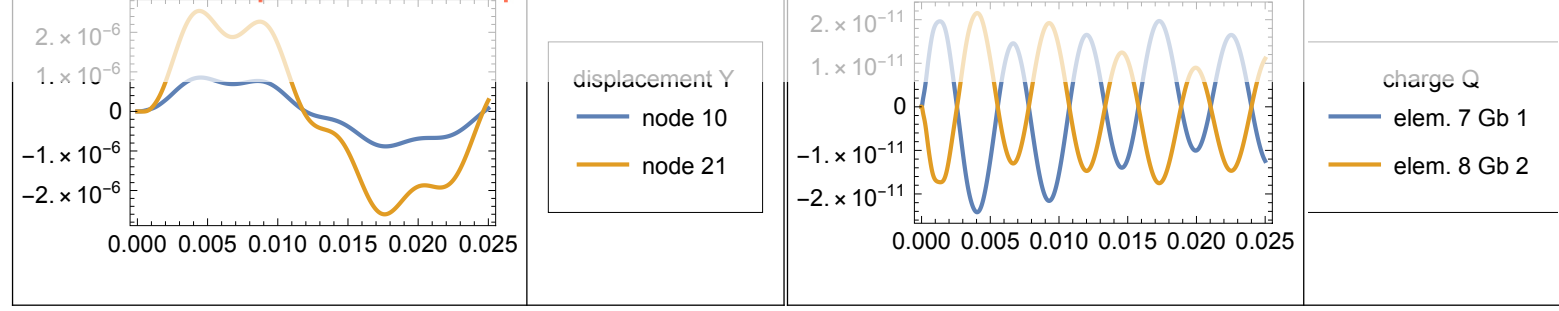

Figure 9: Excitation by electric potential on actuators - displacements and the electric charge

in this analysis the system had zero deformation and zero velocity at the beginning of investigated time.

\subsection{MOR state-space model}

Based on the modal analysis results, a reduced state space model described by equation (15) was created using the first 4 bending eigenfrequencies and their corresponding 
eigenshapes. In the model, the damping of system was defined as in the FEM model. In the dynamic analysis of the reduced state-space model, all excitations which were used in the transient FEM analysis, were considered. Initial deformations, external vertical force located at the right end of the beam structure and electric potential on actuators had to be transformed into modal space. Fig. 10 shows the vertical displacement of the node 21, which was calculated from the full FEM model - blue line and from the reduced state space model - brown rings, considering a system without damping as well as a system with damping. Fig. 11 shows time variation of the vertical displacement of the node 21
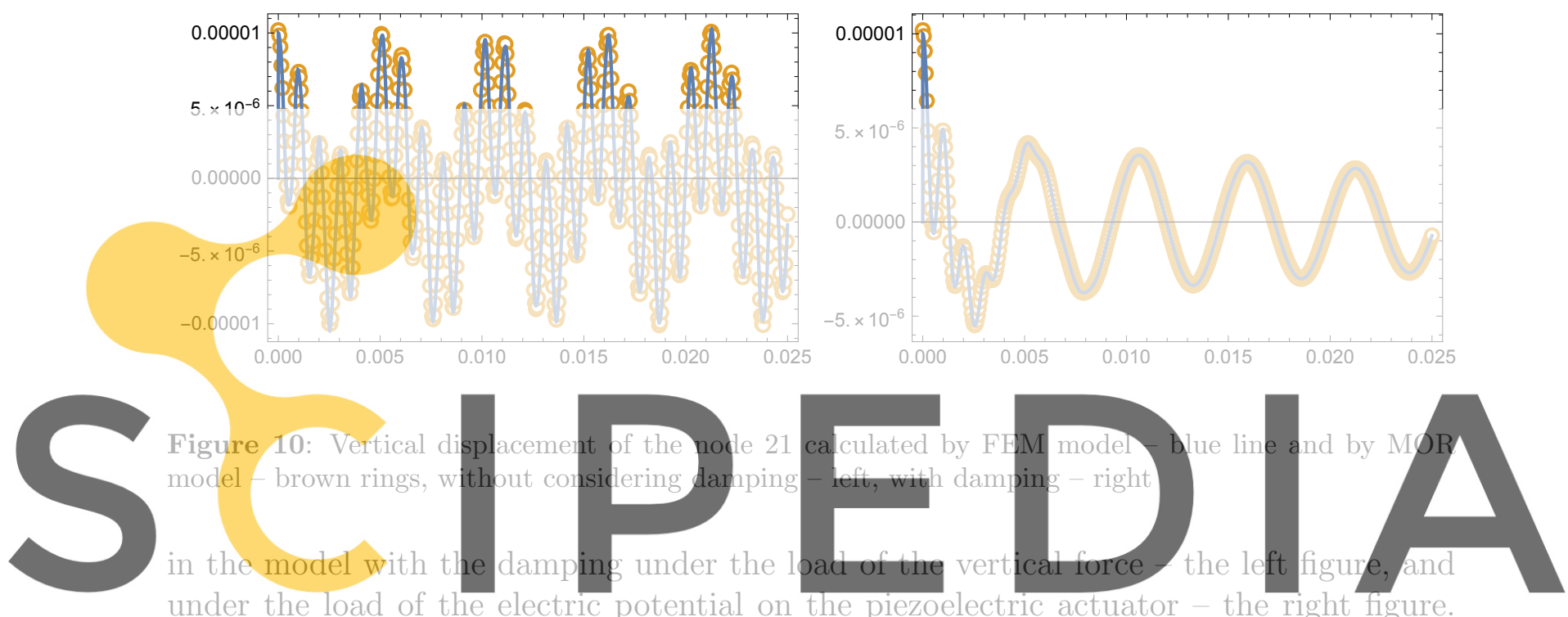

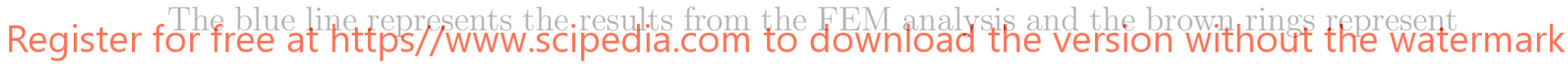
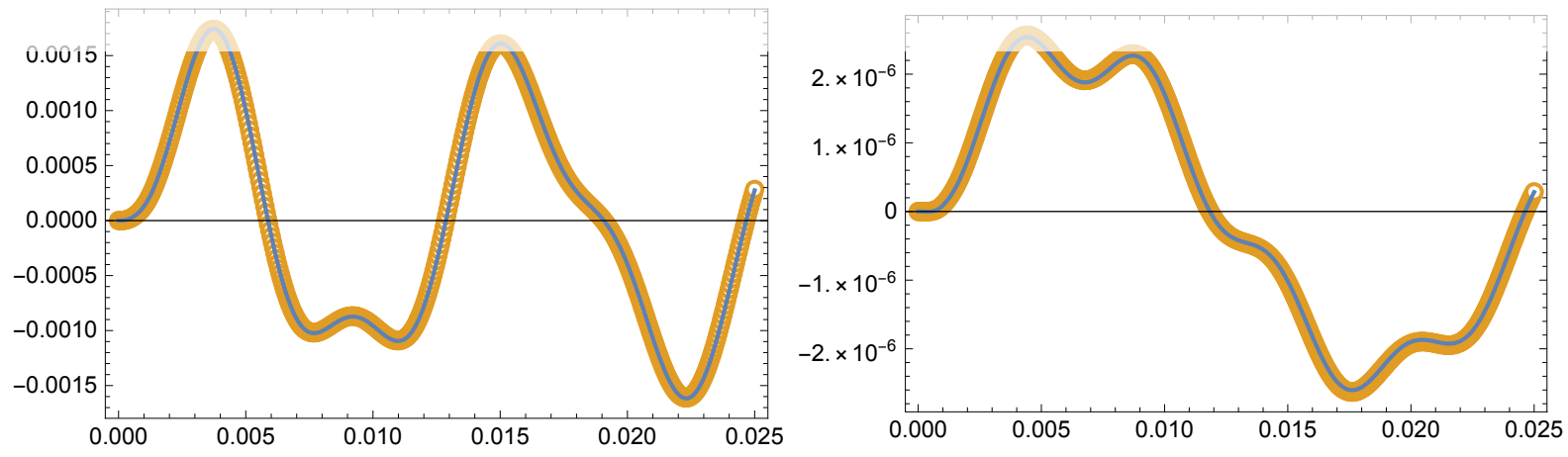

Figure 11: Vertical displacement of the node 21 calculated by FEM model - blue line and by MOR model - brown rings, system loaded by vertical force - left, system loaded by electric potential on the piezoelectric actuator - right

the results from the reduced state-space model. As can be seen from Fig. 10 and 11, MOR 
state-space model very faithfully describes the behavior of the system and the state-space model have significantly fewer degrees of freedom than the full FEM model.

\subsection{Control law}

A reduced state space model was used to design the LQR controller, with a state weight of 50,000 and an input weight of 1 . Based on the parameters thus selected, the gain matrix $\mathbf{K}_{L Q R}$ was calculated and then a feedback state-space model was built. Subsequently, the state-space model with feedback was analyzed, considering the initial conditions defined by static FEM analysis and transformed into modal space. Comparison of the system response, i.e. the vertical deflection of the node 21 for the system without control - blue line and for the system with control - brown line, is shown in Fig. 12 left. The electric

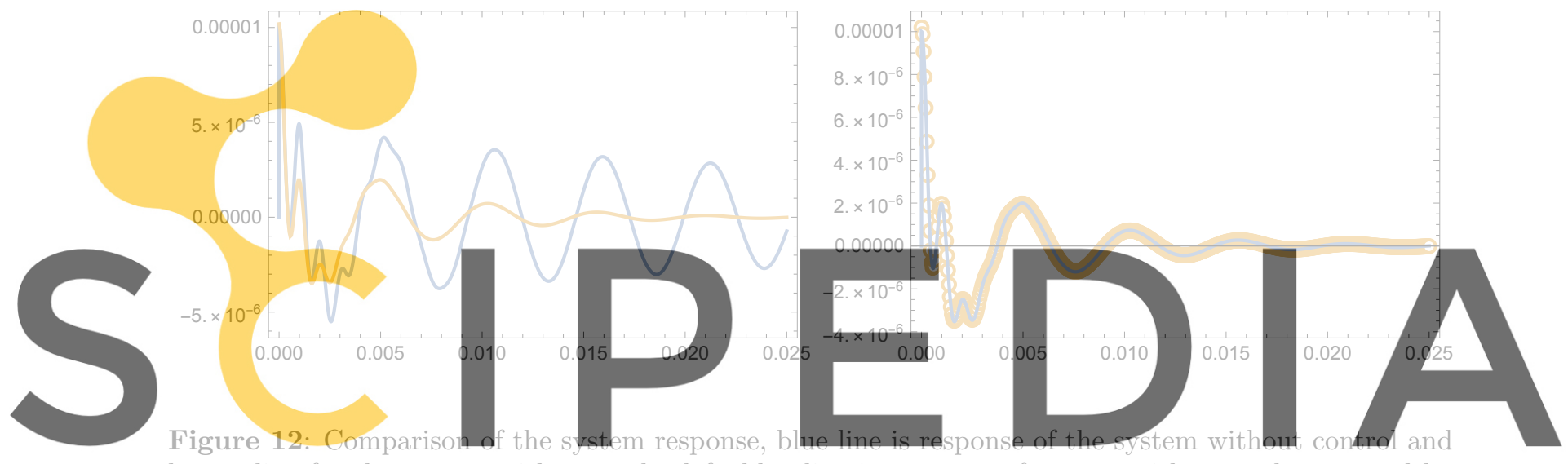

brown line for the system with control - left, blue line is response of system with control computed by

potential on the piezoclectric actuators generated from the linear quadratic controller was determined - time variation of electric potential is shown in Fig. 13. This voltage was

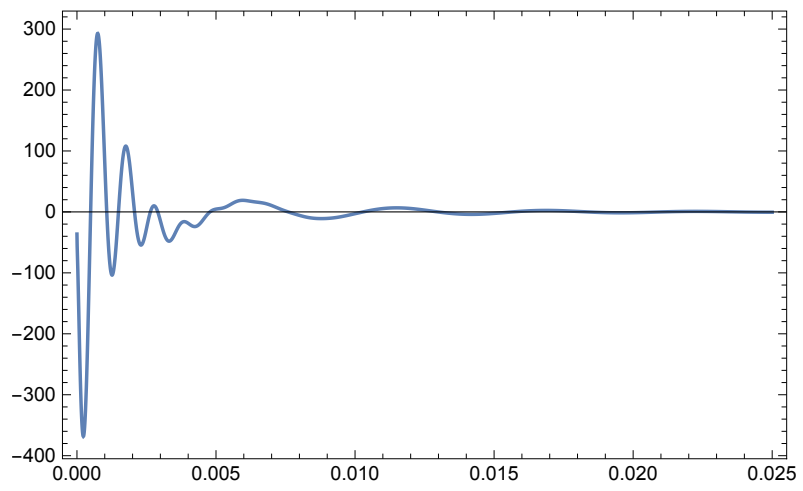

Figure 13: The electric potential on the actuators generated from the linear quadratic controller 
applied as a load to the full FEM model and a transient FEM analysis was performed. A comparison of the results from the FEM model and the MOR model when control is considered is shown in Fig. 12 right, where the blue line represents the displacement of node 21 from the FEM analysis and the brown rings from the MOR analysis.

\section{CONCLUSIONS}

The paper presents finite beam element with core made from functionally graded material and piezoelectric patches placed on the outer surfaces. The derived FEM equations were implemented in the FEM program MultiFEM. In this program, static, modal, harmonic and full transient analysis were performed. Using these analyzes a reduced state-space model of the system was created. MOR state-space model was analyzed using program Mathematica, and the results from the MOR model were compared with the results from the FEM model. The comparison showed a very good agreement between these two models. As a last step, linear quadratic regulator for a reduced state-space model was designed. The results from the MOR model with LQR controller were also compared with the FEM results, where the electric load of piezoelectric actuator was derived from gain matrix and states of system.

\section{ACKNOWLEDGEMENT}

This work was supported by the Slovak Grant Agency: VEGA 1/0081/18, VEGA 1/0416/21, KEGA 011STU-4/2020 and APVV-19-0406.

\section{REFERENCES}

[1] Schwartz, M. Encyclopedia of Smart Materials. John Wiley \& Sons, Inc., (2002).

[2] Dorf, R. C. and Bishop, R. H. Modern Control Systems. Pearson, (2017).

[3] Varadan, V. K., Vinoy, K. J. and Gopalakrishnan, S. Smart Material Systems and MEMS:Design and Development Methodologies. John Wiley \& Sons, Inc., (2006).

[4] Friedland, B. Control System Design An Introduction to State-Space Methods. Dover Publications, Inc., (2005).

[5] Burnett, D.S. Finite Element Analysis: From Concepts to Applications. Addison Wesley Publishing Company, (1987).

[6] Besselink, B. et al., A Comparison of Model Reduction Techniques from Structural Dynamics, Numerical Mathematics and Systems and Control. Journal of Sound and Vibration (2013) 332:4403-4422.

[7] Wolfram Research, Inc. Mathematica, Version 12.2. Publisher: Wolfram Research, Inc., (2020). 